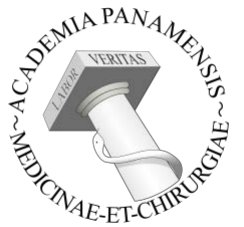

Artículos científicos

Características clínicas y demográficas de pacientes con cirrosis hepática, Hospital Dr. Gustavo Nelson Collado Ríos. Enero - diciembre 2019

[Clinical and demographic characteristics of patients with liver cirrhosis, Hospital Dr. Gustavo Nelson Collado Ríos. January-December 2019]

\title{
Mendieta Ireni
}

Hospital Dr. Gustavo Nelson Collado Ríos. Herrera, Panamá.

\section{Palabras Claves: cirrosis al- cohólica, esteatohepatitis no alcohólica, hepatitis virales}

Keywords: alcoholic cirrhosis, non-alcoholic steatohepatitis, viral hepatitis

Correspondencia a:

Dra. Ireni Mendieta I.

Correo electrónico: irenimendieta@gmail.com

Recibido:19 de enero de 2021 Aceptado: 17 de mayo 2021 Publicado: 15 de jun. 2021

\section{Aspectos bioéticos:}

La autora declara que no existe conflicto de interés alguno asociado en la publicación del manuscrito. Siendo un estudio realizado dentro del programa de residencias médicas, el tiempo para la revisión bibliográfica, la recolección y el análisis de los datos se financió con recursos propios. Esta investigación fue aprobada por el Comité Institucional de Ética e Investigación de la Caja de Seguro Social, Policlínica Manuel Ferrer Valdés.

\section{Resumen}

La cirrosis es un padecimiento irreversible, de elevada mortalidad y de etiología variada, siendo las causas más asociadas: abuso de alcohol, hepatitis por virus $\mathrm{B}$ y $\mathrm{C}$ y hepatopatías autoinmunes. Objetivo Describir las características clínicas y demográficas de los pacientes con cirrosis hepática en el Hospital Gustavo Nelson Collado Ríos. Enero- Diciembre 2019. Metodología Se realizó un estudio observacional, descriptivo y retrospectivo, para lo cual se revisaron 42 expedientes clínicos de pacientes con cirrosis hepática que acudieron a sus citas con el servicio de Gastroenterología en el año 2019. Resultados El grupo de edad con mayor número de casos fue 60-69 años con predominio del género femenino. Los factores asociados más frecuentes fueron diabetes mellitus $2(62 \%)$, sobrepeso $(38 \%)$ obesidad (33\%), hábito de alcohol (19\%), hepatopatías autoinmunes $(12 \%)$ y virus de la hepatitis B (7\%). Se calculó una letalidad de $20 \%$ para esta cohorte, una mediana de sobrevida de 2 años para los pacientes vivos al momento del estudio y una mediana de sobrevida de 18 meses para los pacientes fallecidos por complicaciones de cirrosis en el año 2019 Conclusión en las provincias centrales de Panamá, vemos una tendencia a la disminución de casos de cirrosis por etiología alcohólica y viral, con aumento de enfermedades metabólicas predominantemente en el género femenino que provocan lesión hepática a largo plazo. La cirrosis continúa siendo una enfermedad de alta letalidad; sin embargo con una sobrevida que sobrepasa los 2 años si se mantiene al paciente compensado.

\section{Abstract}

Cirrhosis is an irreversible condition, with high mortality and of varied etiology, the most associated causes being: alcohol abuse, hepatitis $B$ and $C$ virus and autoimmune hepatopathies. Objective To describe the clinical and demographic characteristics of patients with liver cirrhosis at the Gustavo Nelson Collado Ríos Hospital. January- December 2019. Methodology An observational, descriptive and retrospective study was conducted, for which 42 clinical records of patients with liver cirrhosis who attended their appointments with the Gastroenterology service in 2019 were reviewed. Results The age group with the highest number of cases was 60-69 years with a predominance of the female gender. The most frequent associated factors were diabetes mellitus $2(62 \%)$, overweight $(38 \%)$, obesity $(33 \%)$, alcohol habit (19\%), autoimmune liver diseases (12\%) and hepatitis B virus (7\%). A case fatality of $20 \%$ was calculated for this cohort, a median survival of 2 years for patients alive at the time of the study and a median survival of 18 months for patients who died of cirrhosis complications in 2019. Conclusion In the central provinces of Panama, we see a trend towards a decrease in cases of cirrhosis due to alcoholic and viral etiology, with an increase in metabolic diseases predominantly in the female gender that cause long-term liver damage. Cirrhosis continues to be a disease of high lethality; however, with a survival that exceeds 2 years if the patient remains compensated. 


\section{INTRODUCCION}

La cirrosis hepática es una enfermedad crónica del hígado, difusa e irreversible, caracterizada por la presencia de fibrosis que conduce a una alteración de la arquitectura vascular, así como de la funcionalidad hepática [1]. Desde el punto de vista histológico, además de fibrosis extensa, se acompaña de la formación de nódulos de regeneración en el parénquima hepático [1].

Es una importante causa de morbilidad y mortalidad en el mundo. Se estiman ochocientas mil (800 000) muertes cada año a nivel mundial y se encuentra entre las diez principales causas de muerte en Occidente [2]. A pesar de los esfuerzos, las tasas de defunción siguen siendo elevadas, ocupando los primeros lugares a nivel mundial; Moldavia, 91 por cien mil (100 000) habitantes y Hungría, 85 por cien mil (100 000) habitantes, mientras que las cifras más bajas, entre 3 y 5 por cien mil $(100$ 000) habitantes, corresponden a Irlanda, Colombia, Holanda, Singapur, Israel y Noruega [3]. La prevalencia de esta patología es variable según el área geográfica; predomina en el sexo masculino y su pico de incidencia se presenta entre los 40 y 50 años de edad [2].

América no escapa a estas cifras. En la década de 19801989 , las tasas más altas de mortalidad por cirrosis en el mundo, se registraban en México y Chile [4]. Un estudio realizado en Lima, Perú, determinó que la causa más frecuente de esta enfermedad es la ingesta de alcohol $(28 \%)$, seguida de la hepatitis viral B $(15,2 \%)$, hepatitis C $(11,8 \%)$, enfermedades autoinmunes $(9,9 \%)$, esteatohepatitis no alcohólica $(2,5 \%)$ y otros $(0,4 \%)$ [3].

Para desarrollar una enfermedad hepática alcohólica es necesario un consumo diario de alcohol de entre 30-60 g en los varones y de $20-40 \mathrm{~g}$ en las mujeres durante un período mayor a 10 años [5].

Es poco frecuente la hepatitis autoinmune con evolución a cirrosis ya que presenta buena respuesta al tratamiento de remisión; sin embargo, los pacientes que ya tienen cirrosis cuando se diagnostica la enfermedad, tienen un peor pronóstico [6].

El hígado graso no alcohólico y la esteatohepatitis no alcohólica han sido asociadas con lesiones crónicas del hígado que pueden llevar a un cuadro de cirrosis hepática [7]. Los datos actuales respecto a la prevalencia de esta entidad, varían en función de la población estudiada (edad, sexo, raza, comorbilidades), de las diferencias geográficas y de los métodos diagnósticos utilizados. Los resultados de un meta análisis que analizó un total de 86 estudios de 22 países con una muestra de más de ocho millones (8 000000$)$ de individuos muestran una prevalencia global de $25 \%$ [7].

A su vez, la prevalencia de enfermedad hepática grasa no alcohólica es significativamente superior en los pacientes con sobrepeso, obesidad y/o Diabetes Mellitus 2, en comparación con la población general, llegando hasta $70 \%$ [8]. A pesar que existen factores que influyen en la sobrevida de pacientes con cirrosis hepática como la edad, clase funcional y la presencia de complicaciones al momento del diagnóstico (ascitis, ictericia, encefalopatía, hemorragia digestiva, etcétera), la etiología es el principal determinante. Un estudio en México estimó una supervivencia global de los pacientes con cirrosis hepática de 50 \pm 3 meses [8].

Desde el 2011, en el Programa Nacional de Trasplante de la Caja de Seguro Social (CSS) [6] se han realizado 65 trasplantes hepáticos, hasta el 2018. Aproximadamente $40 \%$ a $45 \%$, de los trasplantes hepáticos son secundarios a consumo de alcohol [6]. Sin embargo, aunque el alcohol es el principal detonante de la cirrosis hepática, no es el único factor que desencadena este padecimiento, ya que el $20 \%$ a $25 \%$ de los trasplantados es por etiología viral, específicamente, hepatitis C [6]. El resto de las intervenciones quirúrgicas se han realizado en pacientes con hígado graso, no alcohólico [6].

En cuanto a las hepatitis virales, según el Ministerio de Salud, en Panamá en el año 2016, se registraron 255 casos de hepatitis B y 15 casos de hepatitis C; cifras con tendencia al descenso [7].

La esteatohepatitis secundaria a enfermedades metabólicas puede progresar hacia cirrosis en un $20 \%$ de los pacientes y tiene una tasa de mortalidad superior al $10 \%$ entre los 10 y 15 años tras el diagnóstico [9].

Se describe que la enfermedad hepática grasa no alcohólica pronto será la enfermedad del hígado más frecuente, junto con la cirrosis de origen alcohólico [10-12].

El objetivo general de este trabajo es describir las características clínicas y demográficas de pacientes con cirrosis hepática en el Hospital Gustavo Nelson Collado Ríos. Enero- Diciembre 2019. Como objetivos específicos planteamos calcular la letalidad y la mediana de sobrevida de esta cohorte de pacientes con cirrosis hepática.

\section{MATERIALES Y MÉTODOS}

Se realizó un estudio observacional, descriptivo y retrospectivo. El área de estudio fue el Hospital Dr. Gustavo Nelson Collado Ríos en Chitré, provincia de Herrera, Panamá. La fuente primaria de esta investigación fueron los expedientes clínicos de pacientes con cirrosis hepática.

El universo de estudio lo constituyó 42 pacientes procedentes de Herrera, Los Santos, Coclé y Veraguas. Se trabajó con la totalidad de casos.

Criterios de inclusión: Pacientes con 20 años de edad y 
más con diagnóstico de cirrosis hepática, procedentes de los expedientes clínicos; sin embargo, se protegió la inprovincias centrales, atendidos por el servicio de gas- formación con la firma de un acuerdo de confidencialidad troenterología del Hospital Dr. Gustavo Nelson Collado por la investigadora.

Ríos en enero a diciembre 2019; pacientes fallecidos por complicaciones de cirrosis hepática en dicha institución y periodo del estudio.

Criterios de exclusión: Pacientes con sospecha de cirrosis hepática sin confirmación por ultrasonido; pacientes con expediente incompleto; pacientes fallecidos por causas o complicaciones distintas a cirrosis hepática; pacientes que no acudieron o perdieron al menos una de sus citas durante el estudio.

Para el análisis descriptivo se utilizaron frecuencias absolutas y relativas. El análisis estadístico fue realizado en EPI Info, versión 7.2 y Microsoft Excel.

\section{RESULTADOS}

Para la recolección de datos se solicitó al departamento de estadística, las hojas de registro diario de la consulta externa de gastroenterología para obtener la cifra de pacientes con diagnóstico de cirrosis hepática atendidos de enero a diciembre 2019; así mismo se obtuvo el número de fallecidos por complicaciones de cirrosis hepática. Luego, con la autorización y clave del programa SIS se ingresó al expediente electrónico para tener acceso a la historia clínica de los pacientes.

Se utilizaron las siguientes variables: sexo, edad, procedencia, hábito de alcohol, sobrepeso, obesidad, diabetes mellitus 2 , hepatitis autoinmune, hepatitis viral $\mathrm{B}$, hepatitis viral $\mathrm{C}$, letalidad y sobrevida.

Los datos fueron obtenidos en el mes de septiembre de 2020 mediante el formulario de recolección aprobado. No se utilizó consentimiento informado ya que se trabajó con

En relación al sexo, predominó el género femenino con $62 \%$ en comparación con el género masculino con $38 \%$.

El grupo etario con mayor cantidad de pacientes con cirrosis hepática fue de $60-69$ años con $38 \%$ y la provincia

Tabla No 1. Pacientes con cirrosis hepática según antecedente de diabetes mellitus 2 .

\begin{tabular}{cccc}
\hline $\begin{array}{c}\text { Diabetes } \\
\text { Mellitus 2 }\end{array}$ & $\begin{array}{c}\text { Cantidad de } \\
\text { pacientes }\end{array}$ & $\%$ & IC 95\% \\
\hline Total & 42 & 100 & - \\
Sí & 26 & 62 & $45,6-76,4$ \\
No & 16 & 38 & $23,5-54,3$ \\
\hline
\end{tabular}

En la investigación se encontró que el factor mayormente asociado a cirrosis hepática fue diabetes mellitus 2 con $62 \%$ IC $(45,6-76,4)$. Fuente: Expedientes clínicos. Consulta externa de Gastroenterología, Hospital Gustavo Nelson Collado Ríos. CSS. Chitré 2019.

Gráfica No 1. Pacientes con cirrosis hepática según grupo de edad.

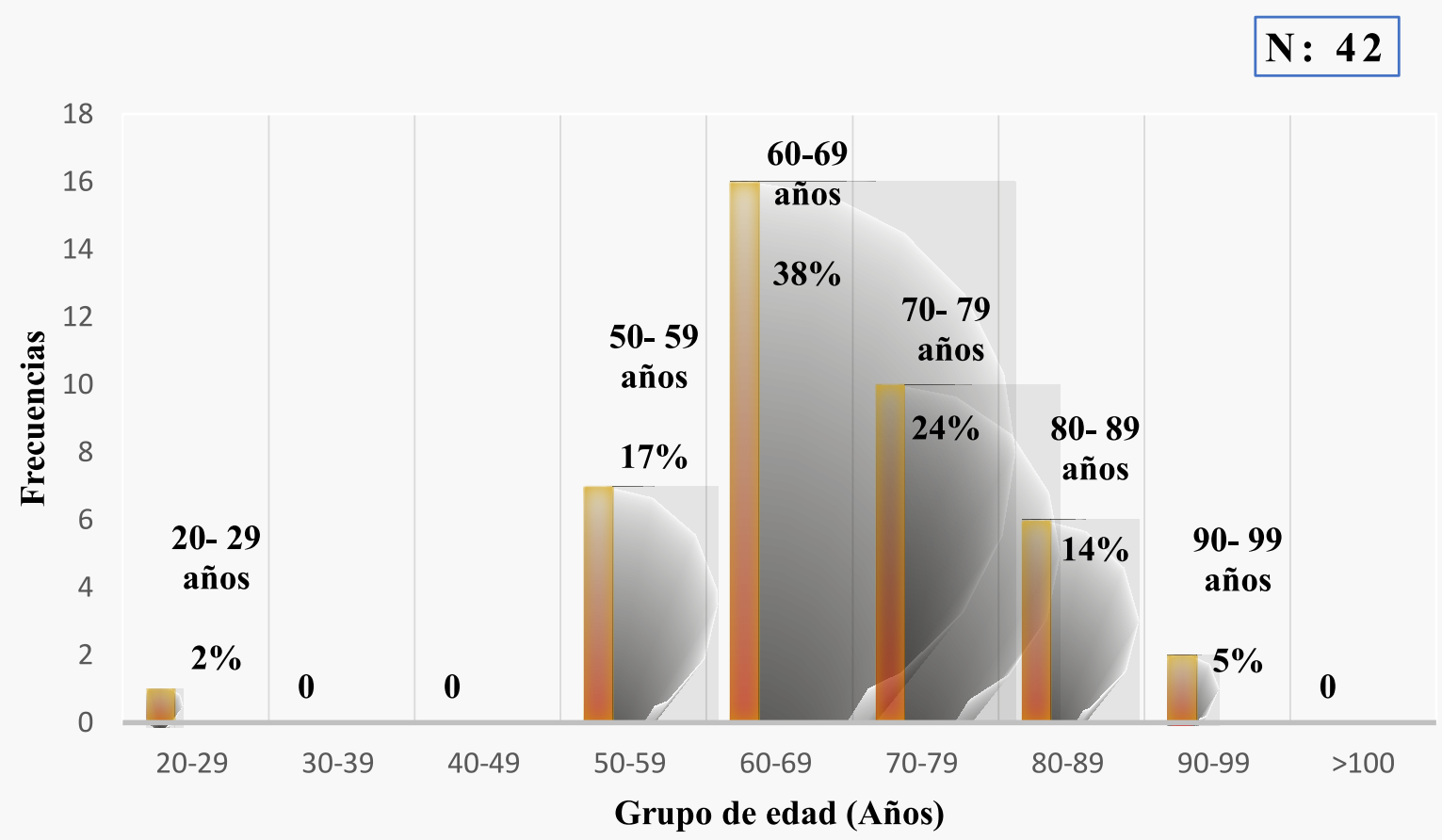

CSS. Chitré 2019. El grupo etario con mayor cantidad de pacientes con cirrosis hepática fue de 60-69 años con 38\% IC $(21,4-57,1)$ y la provincia que registró el mayor porcentaje de casos fue Herrera con $48 \%$ IC $(32,0-63,5)$. Fuente: Expedientes clínicos. Consulta externa de Gastroenterología, Hospital Gustavo Nelson Collado Ríos. 
Gráfica No 2. Pacientes con cirrosis hepática según índice de masa corporal.

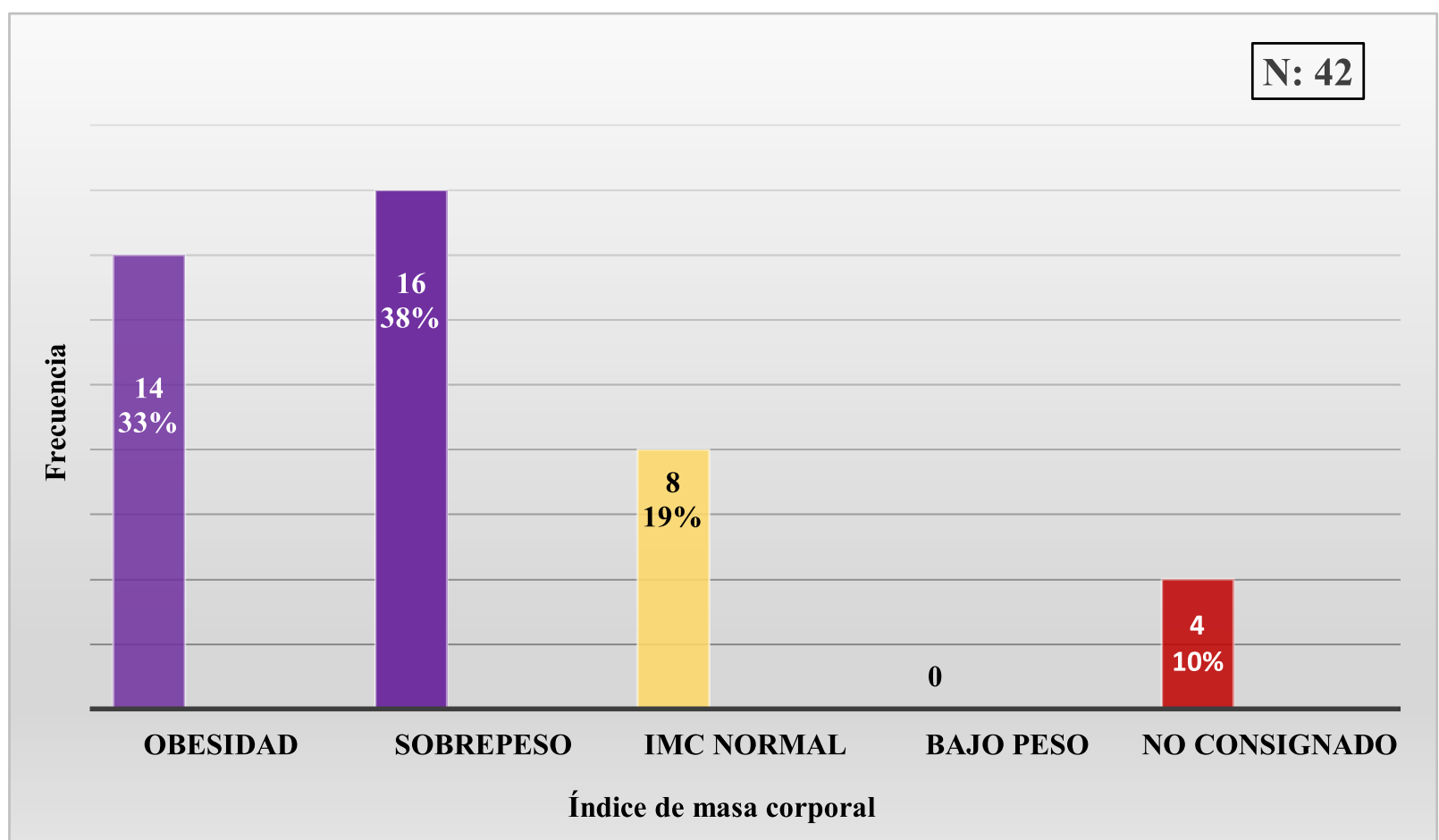

Seguido de diabetes mellitus 2, los factores más frecuentes fueron: sobrepeso con $38 \%$ IC $(22,9-53,2)$ y obesidad con $33 \%$ IC $(21,0-50,9)$. Fuente: Expedientes clínicos. Consulta externa de Gastroenterología, Hospital Gustavo Nelson Collado Ríos. CSS. Chitré 2019.

que registró el mayor porcentaje de casos fue Herrera con $48 \%$. ( Ver gráfica No 1).

En la investigación se encontró que el factor mayormente asociado a cirrosis hepática fue diabetes mellitus 2 con $62 \%$ (Ver tabla No 1).

Seguido de diabetes mellitus 2, los factores más frecuentes fueron: sobrepeso con $38 \%$ y obesidad con $33 \%$ ). (Ver gráfica No 2).

Los pacientes con hábito de alcohol representaron el 19\% (Ver tabla No 2). La enfermedad autoinmune representó el $12 \%$ y la hepatitis viral B el $7 \%$ No hubo casos de hepatitis viral C. (ver tabla No 3).

Se obtuvo una mediana de sobrevida para los pacientes vivos al momento del estudio, a partir de la fecha del diagnóstico hasta diciembre de 2019, de 24 meses (2 años). (Ver gráfica No 4).

Además, se calculó una mediana de sobrevida para los pacientes fallecidos en el año 2019 por complicaciones de cirrosis hepática de 18 meses (1 año y 6 meses).

Del total de casos, 5 (12\%) pacientes fueron diagnosticados con cirrosis hepática durante el periodo enero-diciembre 2019 y de ellos 1 (2\%) paciente falleció. Con estos datos se calculó una tasa de letalidad de $20 \%$.

Tabla No 2. Pacientes con cirrosis hepática según hábito de alcohol.

\begin{tabular}{cccc}
\hline $\begin{array}{c}\text { Hábito de } \\
\text { Alcohol }\end{array}$ & $\begin{array}{c}\text { Cantidad de } \\
\text { pacientes }\end{array}$ & $\%$ & IC 95\% \\
\hline Total & 42 & 100 & - \\
Sí & 8 & 19 & $8,6-34,1$ \\
No & 34 & 81 & $65,8-91,4$ \\
\hline
\end{tabular}

Los pacientes con hábito de alcohol representaron el 19\% IC (8,6-34,1). Fuente: Expedientes clínicos. Consulta externa de Gastroenterología, Hospital Gustavo Nelson Collado Ríos. CSS. Chitré 2019.

Tabla No 3. Pacientes con cirrosis hepática según diagnóstico de hepatitis autoinmune y/o hepatitis viral.

\begin{tabular}{lccc}
\hline Diagnóstico & $\begin{array}{c}\text { Cantidad de } \\
\text { pacientes }\end{array}$ & $\%$ & IC 95\% \\
\hline Total & 42 & 100 & - \\
Hepatitis & 5 & 12 & $3,9-25,6$ \\
autoinmune & & 7 & $2,6-22,6$ \\
Hepatitis B & 3 & 0 & - \\
Hepatitis C & 0 & $(3,9-25,6)$ y \\
La enfermedad autoinmune representó el & $12 \%$ IC & \\
la hepatitis viral B el 7\% IC (2,6-22,6). No hubo casos de \\
hepatitis viral C. Fuente: Expedientes clínicos. Consulta \\
externa de Gastroenterología, Hospital
\end{tabular}


Gráfica No 4. Sobrevida en meses de los pacientes vivos con cirrosis hepática a partir de la fecha del diagnóstico hasta diciembre 2019.

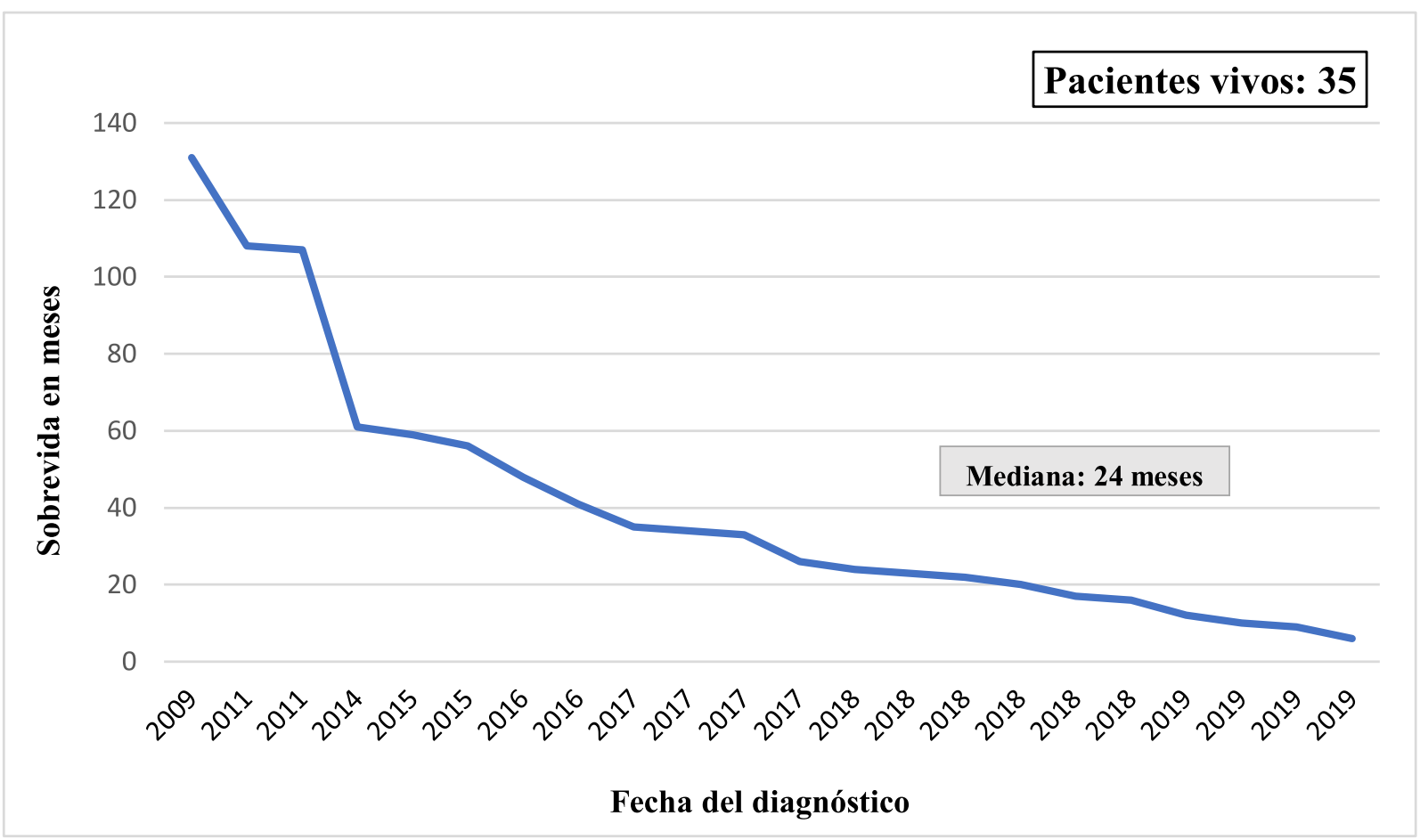

Se obtuvo una mediana de sobrevida para los pacientes vivos al momento del estudio, a partir de la fecha del diagnóstico hasta diciembre de 2019, de 24 meses (2 años). Fuente: Expedientes clínicos. Consulta externa de Gastroenterología, Hospital Gustavo Nelson Collado Ríos. CSS. Chitré 2019.

\section{DISCUSIÓN}

El mayor número de casos con cirrosis hepática fue en el género femenino con $62 \%$ y el grupo etario de $60-69$ años con $38 \%$; sin embargo, resultados distintos fueron determinados en México, con un promedio de edad de 55.7 años y con predominio del género masculino [13-15].

Estudios previos en Perú y México describen a la ingesta de alcohol como la causa más frecuente de cirrosis hepática $[13,15]$. En contraparte, el estudio mostró que el factor mayormente asociado fue diabetes mellitus $2(62 \%)$, seguido de sobrepeso (38\%) y obesidad (33\%).

Herrera registró la mayor cantidad de casos con $48 \%$ y a pesar de ser la principal región productora de alcohol en Panamá [16], se obtuvo una cantidad baja de pacientes cirróticos con hábito de alcohol.

Los resultados del estudio reflejan el aumento de enfermedades metabólicas en Panamá. Sin duda alguna, la diabetes, el sobrepeso y la obesidad se han convertido en una pandemia. En los inicios del presente siglo XXI, las cifras eran aproximadamente de 150 millones de personas diabéticas; pero incluso se espera que afecte a 380 millones para el 2025, con un aumento mayor en la población de 45 a 64 años en los países tercermundistas [17].
La segunda etiología de cirrosis hepática en los estudios en Perú y México, [13-15] fueron las hepatitis virales $B$ y C. Sin embargo, en provincias centrales de Panamá se obtuvo sólo un $7 \%$ de casos con hepatitis B que evolucionaron a cirrosis. A pesar que en Panamá se estima que un $20 \%$ de los infectados por hepatitis C desarrolla cirrosis hepática, no hubo casos de hepatitis $C$ en nuestra cohorte, lo cual pudiese explicarse por la tendencia al descenso de casos a partir del 2016 [7] y a la mayor prevalencia de hepatitis $B$ en la población general; además de la labor que realiza la clínica de hepatitis virales en el Complejo Hospitalario Dr. Arnulfo Arias Madrid para evitar que los pacientes con diagnóstico de hepatitis viral progresen a cirrosis [17].

En relación al diagnóstico de hepatitis autoinmune, se obtuvo un $12 \%$, similar a Perú con 9,9\% [3].

La letalidad calculada de esta cohorte para el 2019 fue de $20 \%$. No se encontraron estudios sobre letalidad; sin embargo, en México se obtuvo una mortalidad hospitalaria de $23.5 \%$ [5].

Por último, de acuerdo con la escala de severidad de cirrosis hepática CHILD PUGH, se estima que los pacientes con cirrosis compensada tienen una sobrevida a dos años de $85 \%$; sin embargo aquellos pacientes descompensados tienen una sobrevida a dos años de 35\% [17]. En la investigación se calculó una mediana de sobrevida 
de 2 años para los pacientes vivos al momento del estu- do la progresión de la enfermedad y mejorando la calidad dio y una mediana de sobrevida de 18 meses para los de vida de los pacientes.

pacientes fallecidos por complicaciones de cirrosis hepática, muy acorde a la escala pronóstica.

\section{CONCLUSIONES}

En las provincias centrales de Panamá, vemos una tendencia a la disminución de casos de cirrosis hepática por etiología alcohólica y viral, con aumento de enfermedades metabólicas, predominantemente en el género femenino que provocan lesión hepática a largo plazo.

La cirrosis continúa siendo una enfermedad de alta letalidad; sin embargo, con una sobrevida que sobrepasa los 2 años si se mantiene al paciente compensado.

El diagnóstico temprano es fundamental porque ofrece la oportunidad de tratar las causas subyacentes, previnien-

\section{REFERENCIAS}

[1] Tsochatzis, E., Bosch, J., \& Burroughs, A. (28 de 01 de 2014). Liver cirrhosis. Lancet, 383(9930), 174961. doi: 10.1016/S0140-6736(14)60121-5.

[2] Torreblanca, A., \& Fonseca, L. (12 de 2014). Caracterización de los pacientes con cirrosis hepática atendidos en Las Tunas. Revista electrónica Dr Zoilo E. Marinello Vidaurreta, 39(12). Obtenido de http:// revzoilomarinello.sld.cu/index.php/zmv/article/view/ $121 / 209$

[3] Bustios, C., Davalos, M., Roman, R., \& Zumaeta, E. (2007). Características Epidemiológicas y Clínicas de la Cirrosis Hepática en la Unidad de Hígado del HNERM. Revista de Gastroenterología del Perú, 27(3), 238-245. Obtenido de http://www.scielo.org.pe/ scielo.php?pid=S1022$51292007000300003 \&$ script=sci abstract

[4] Bosetti, C., Levi, F., Lucchini, F., Zatonski, W., Negri, E., \& La Vecchia, C. (16 de 02 de 2007). Worldwide mortality from cirrhosis: an update to 2002. Hepatology, 46(5), 827-39. doi:10.1016/j.jhep.2007.01.025.

[5] Rosales, José Miguel. 2018. Portal de la Asociación Catalana de pacientes hepáticos (citado el 21 de septiembre de 2020). Dirección de URL: https://asscat-hepatitis.org/

[6] Lasso. (09 de Septiembre de 2018). CSS bate récord de trasplante de hígado en 2018. Panamá América (citado el 21 de septiembre de 2020). Dirección de URL: https://www.panamaamerica.com.pa/sociedad/ video-css-bate-record-de-trasplante-de-higado-en2018-1115652

[7] Ruiloba. 2017. Portal del Ministerio de Salud de la República de Panamá. Centro de Prensa (citado el 21 de septiembre de 2020). Dirección de URL: http://
Este estudio en pacientes con cirrosis hepática en las provincias de Herrera, Los Santos, Veraguas y Coclé proporciona una idea de la magnitud de la enfermedad y abre nuevas líneas de investigación y prevención.

\section{Limitaciones}

Debido a las restricciones por la pandemia de coronavirus 2019, no se aplicó una encuesta para obtener cantidad, frecuencia y tipo de alcohol.

\section{Agradecimientos}

Asesores: Dra. Bárbara Hurtado - Epidemiología. Dr. Walter Bernal- Gastroenterología.

www.minsa.gob.pa/noticia/minsa-se-une-la-campana-del-dia-mundial-de-la-hepatitis-bajo-el-lema-elimine-la-hepatitis

[8] EASL (2015). Clinical Practice Guidelines: Autoimmune hepatitis. Journal of Hepatology vol. 63: 9711004.

[9] Younossi, Z., Koenig, A., Abdelatif, D., Fazel, Y., Henry, L., \& Wymer, M. (22 de 02 de 2016). Global epidemiology of nonalcoholic fatty liver disease-Meta-analytic assessment of prevalence, incidence, and outcomes. Hepatology, 64(1), 73-84. doi:10.1002/ hep.28431.

[10] Aller, R., Fernández-Rodríguez, R., laconoc, O., \& al, e. (2018). Documento de consenso. Manejo de la enfermedad hepática grasa no alcohólica (EHGNA). Guía de práctica clínica. Elsevier, 12(003), 1-22. doi:org/10.1016/j.gastrohep.2017.12.003.

[11] Sampedro. 2018. Portal de la Asociación Catalana de pacientes hepáticos (citado el 21 de septiembre de 2020). Dirección de URL: https://asscat-hepatitis.org/una-de-cada-cuatro-personas-presenta-enfermedad-hepatica-grasa-no-alcoholica/

[12] Rodríguez, H., Jacobo, J., Castañón, M., Arámbula, M., \& Martínez, G. (2002). Supervivencia de pacientes con cirrosis hepática en el Hospital General Regional del IMSS, Durango. Gac Méd Méx, 138(4), 325-30. Obtenido de https://www.anmm.org.mx/ bgmm/1864_2007/2002-138-4-325-330.pdf

[13] Zubieta, J., Gómez, R., Rodríguez, K., Ariza, N., \& Toloza. (2017). Mortalidad hospitalaria en pacientes cirróticos en un hospital de tercer nivel. Revista de Gastroenterología de México, 203-209. doi.org/ 10.1016/j.rgmx.2016.10.002.

[14] Olarte, Julio. 2014. Portal del Instituto Nacional de 
Estadística y Censo. Contraloría General de la República de Panamá (citado el 21 de septiembre de 2020). Dirección de URL: https://www.contraloria.gob.pa/INEC/Publicaciones/Publicaciones.aspx?ID_SUBCATEGORIA=15\&ID_PUBLICACION=716\&ID_IDIOMA=1\&ID_CATEGORIA $=4$

[15] Pereira, O. (2012). Diabesidad: una epidemia del siglo XXI. MEDISAN. De http://scielo.sld.cu/scielo.php? script=sci_arttext\&pid=S1029-30192012000200018
[16] Iglesias, Rebeca. 2017. Portal de la Caja de Seguro Social (citado el 21 de septiembre de 2020). Dirección de URL: http://www.css.gob.pa/web/13-junio2017hh.html

[17] Meijide, H. (2014). Slideshare. Obtenido de Clasificación pronóstica de la hepatopatía: https://es.slideshare.net/Juhelemo/child-pugh 